\title{
Role of Coflex as an Adjunct to Decompression for Symptomatic Lumbar Spinal Stenosis
}

\author{
Naresh Kumar ${ }^{1}$, Siddarth M Shah ${ }^{2}$, Yau Hong $\mathrm{Ng}^{2}$, \\ Vinodh Kumar Pannierselvam ${ }^{2}$, Sudeep DasDe ${ }^{2}$, Liang Shen ${ }^{3}$ \\ ${ }^{1}$ University Spine Centre, National University Hospital, Singapore, Singapore \\ ${ }^{2}$ Department of Orthopaedic Surgery, National University Hospital, Singapore, Singapore \\ ${ }^{3}$ Biostatistics Unit, Yong Loo Lin School of Medicine, National University of Singapore, Singapore, Singapore
}

Study design: Prospective cohort study.

Purpose: To assess whether additional implantation of Coflex following spinal decompression provided better clinical outcomes compared to decompression alone for symptomatic lumbar spinal stenosis (LSS) and to determine whether improvement in clinical outcomes correlated with changes in the radiological indices studied.

Overview of Literature: Literature on benefits of additional Coflex implantation compared to decompression alone for symptomatic LSS is limited.

Methods: Patients with symptomatic LSS who met the study criteria were offered spinal decompression with Coflex implantation. Those patients who accepted Coflex implantation were placed in the Coflex group ( $n=22$ ); while those opting for decompression alone, were placed in the comparison group $(\mathrm{n}=24)$. Clinical outcomes were assessed preoperatively, six-months, one-year and two-years postoperatively, using the Oswestry disability index, $100 \mathrm{~mm}$ visual analogue scale (VAS)-back pain and VAS-leg pain, and short form36 (SF-36). Radiological indices (disc height, foraminal height and sagittal angle) were assessed preoperatively, six months, one year, and two years postoperatively.

Results: Both groups showed statistically significant $(p<0.001)$ improvement in all the clinical outcome indicators at all points in time as compared to the preoperative status. However, improvement in the Coflex group was significantly greater $(p<0.001)$ than the comparison group. Changes in the radiological indices did not correlate significantly with the improvement in clinical outcome indicators. Conclusions: Additional Coflex implantation after spinal decompression in symptomatic LSS offers better clinical outcomes than decompression alone in the short-term. Changes in radiological indices do not correlate with the improvements in clinical outcomes after surgery for symptomatic LSS.

Keywords: Spinal stenosis; Decompression; Interspinous process device; Coflex

\section{Introduction}

Lumbar spinal stenosis (LSS) is a common cause of back and leg pain in the elderly, often resulting in significant disability and impaired quality of life [1]. The prevalence of LSS ranges from $1.7 \%$ to $8 \%$ in the general population [2], with a peak incidence in the fifth to seventh decade of life. It is also the most common indication for spinal

Received Nov 25, 2012; Revised Feb 17, 2013; Accepted Feb 17, 2013

Corresponding author: Naresh Kumar

University Spine Centre, University Orthopaedics, Hand \& Reconstructive Microsurgery Cluster,

1E Kent Ridge Road, NUHS Tower Block, Level 11, Singapore 119228, Singapore

Tel: +65-67725611, Fax: +65-67780720, E-mail: dosksn@nus.edu.sg 
Table 1. Inclusion and exclusion criteria

\section{Inclusion criteria}

Patients with symptomatic degenerative lumbar spinal stenosis presenting with back and leg pain

Radiological correlation with clinical presentation

Failure of at least six months of conservative treatment including one or more of the following: analgesia, physiotherapy and acupuncture

Patient's leg symptoms are partially relieved by forward flexion

\section{Exclusion criteria}

Caudaequina syndrome

Previous surgery at the level in question

Spondylolisthesis higher than Meyerding Grade I

Spinal stenosis at $>2$ levels

Spinal stenosis involving the $L 5 / S 1$ segment

De novo scoliosis (Cobb's angle $>15^{\circ}$ )

Spinal infection

Malignant spinal conditions

Positive nerve root tension signs

Instability on flexion/extension lateral radiographs greater than

Meyerding Grade I surgery in the old age population [3]. Back pain in LSS may be due to 1) disc degeneration with secondary facet hypertrophic arthropathy and instability, 2) mechanical compression of nerves by bone, ligament or disc material, and 3) biomechanical mediators of inflammation or pain [4]. Ogikubo et al. [5] showed that there is a direct relationship between the cross sectional area of the dural sac at the most constricted spinal level (mCSA) and the severity of leg and back pain. The smaller the mCSA, the greater is the severity of leg and back pain [5]. Spinal decompression relieves the local stenotic pathology and has been shown to improve the symptoms of back and leg pain after surgery $[6,7]$. There are several studies showing superior results after surgery compared to conservative treatment [7-11] for symptomatic LSS. In the absence of progressive neurological deficit or cauda equina syndrome, surgical options are considered electively when conservative measures fail to relieve the patient's symptoms [12]. Spinal fusion is generally considered when there is preoperative or intraoperative evidence of spinal instability [3,13-15].

The symptoms of LSS are typically worsened on extension and relieved on flexion [16-20]. This has been attributed to the narrowing of the spinal canal and intervertebral foramen on extension, resulting in neural compression. Biomechanical studies have shown that the use of interspinous spacers (ISPs) significantly prevents narrowing of the lumbar spinal canal and intervertebral foramen in extension [21]. While these devices reduce flexion-extension range at the instrumented level, axial rotation and lateral bending at that level and range of motion at the adjacent levels remain unaffected [22]. Additionally, studies have shown that ISPs reduced the intradiscal pressure and stabilized the implanted level $[23,24]$.
Presently, ISPs are being used to address a wide-range of clinical indications like degenerative spinal stenosis, discogenic low back pain, facet syndrome, disc herniation and spinal instability [25].

Coflex (formerly 'Interspinous U'; Paradigm Spine, LCC, New York, NY, USA) is a dynamic ISP. It is a Ushaped, compressible device that can be implanted between the spinous processes after decompression. While there is data comparing the results of Coflex with spinal fusion [26,27], information on its role as an adjunct to lumbar decompression is limited. Thus, the aims of our study are 1) to assess whether additional implantation of Coflex following spinal decompression provided better clinical outcomes compared to spinal decompression alone and 2) to determine whether improvement in clinical outcomes correlated with changes in the radiological indices studied.

\section{Materials and Methods}

A prospective cohort study was conducted at our institute after Institutional Review Board's (IRB) approval. Patients with symptomatic LSS, fulfilling the criteria listed in Table 1 were identified. All patients were offered spinal decompression with Coflex implantation at the affected level(s). Those patients who accepted Coflex implantation were placed in the study group (Coflex group); while the comparison group comprised of those opting for decompression alone. Between May 2007 and July 2009, 46 consecutive patients were recruited in the study-22 in the Coflex group $(n=22)$ and 24 in the comparison group $(n=24)$. Our senior author operated upon all the patients (N.K.).

The mean age of the patients in the Coflex and the comparison group was 57.9 years (range, $40-74$ years) and 
Table 2. Demographic and baseline clinical data of the two groups

\begin{tabular}{lcc}
\multicolumn{1}{c}{ Variable } & Coflex group (n=22) & Comparison group (n=24) \\
Mean age (range, yr) & $57.9(40-74)$ & $61.8(49-78)$ \\
Sex (males:females) & $13: 9$ & $14: 10$ \\
\hline Preoperative ODI score & $51.73 \pm 7.23$ & $49.58 \pm 13.86$ \\
\hline Preoperative VAS (back pain) score & $60.86 \pm 6.39$ & $54.38 \pm 13.90$ \\
\hline Preoperative VAS (leg pain) score & $63.45 \pm 8.98$ & $57.42 \pm 9.96$ \\
\hline Preoperative SF-36 score & $22.914 \pm 3.31$ & $27.329 \pm 7.16$ \\
\hline
\end{tabular}

ODI, Oswestry disability index; VAS, visual analogue scale; SF-36, short form-36.

61.8 years (range, $49-78$ years; $p=0.127$ ), respectively. The male:female ratio in the respective groups was $13: 9(n=22$, Coflex group) and 14:10 ( $\mathrm{n}=24$, comparison group, $p=0.958$ ). Two patients in the Coflex group and one in the comparison group underwent two level surgeries (L3/4 and $\mathrm{L} 4 / 5)$. Rest of the patients underwent one level surgery (L4/5). All patients were followed up for two years postoperatively and none of them was lost to follow-up. The preoperative data in the two groups is illustrated in Table 2.

\section{Surgical technique}

Patients were operated under general anaesthesia and in prone position. Microscopic interlaminar decompression was performed through a standard posterior midline approach in all the patients. Partial laminotomy, excision of hypertrophic ligamentum flavum and undercutting facetectomy were done to decompress the involved stenotic level(s). The Coflex group underwent additional implantation of the Coflex ISP at the involved level(s) through the same surgical incision. In order to implant the device, the supraspinous ligament was dissected subperiosteally, elevated and hinged on the superior spinous process. The interspinous ligament was dissected and excised. The implant was inserted between the adjacent spinous processes and the flanges were crimped as per the manufacturer's instructions, so that it was seated snugly. Satisfactory placement of the implant and adequate segmental sagittal alignment were ensured under image intensifier guidance.

\section{Postoperative care}

All the patients in both groups received similar postoperative care. They were allowed to ambulate as tolerated from the next postoperative day. Early return to activities was encouraged after surgery.

\section{Clinical outcome assessment}

Clinical outcome scores were assessed preoperatively, six months, one year and two years postoperatively using the Oswestry disability index (ODI), $100 \mathrm{~mm}$ visual analogue scale (VAS)-back pain, VAS-leg pain, and short form-36 (SF-36).

\section{Radiological assessment}

Standardized, erect antero-posterior and lateral lumbosacral radiographs, taken preoperatively and immediate, six months, one year and two years postoperatively were assessed by a blind observer, who did not participate in surgery and took all the measurements thrice (SDD) using the GE Centricity Enterprise Web (ver. 3.0, Little Chalfont, Buckinghamshire, UK, 2006). Disc height was calculated by taking the mean of the anterior and posterior disc heights at the level in question. Anterior disc height was calculated as the distance between the anterior inferior corner of the upper vertebral body and the anterior superior corner of the lower vertebral body. Likewise, posterior disc height was calculated as the distance between the posterior inferior corner of the upper vertebral body and the posterior superior corner of the lower vertebral body. The foraminal height was measured as the maximum distance between the inferior margin of the pedicle of the superior vertebra and the superior margin of the pedicle of the inferior vertebra. Sagittal angle was measured by determining the angle subtended between the lines drawn parallel to the superior endplate of the cephalad vertebra and the inferior endplate of the caudad vertebra. Implant subsidence was calculated on serial 
postoperative lateral radiographs by assessing the change in the magnitude of the interspinous line at the affected level. Interspinous line was defined as the line joining the anterior superior edge of the cephalad spinous process to the anterior superior edge of the caudad spinous process (Fig. 1).

\section{Statistical analysis}

Statistical analysis was performed using the Statistical Package for Social Sciences (ver. 19, SPSS Inc., Chicago, IL, USA). A paired t-test was performed to compare the six months, one year and two years ODI, VAS (back pain and leg pain), and SF-36 scores with the respective preoperative scores in both groups. Bonforroni technique was used to adjust for multiple comparisons. Mixed model analysis was performed to compare the improvement in ODI, VAS-back pain and leg pain, and SF-36 scores between the Coflex group and comparison group. Unstructured variance-covariance was used and the baseline was included in the model for adjustment. The influence of duration of follow-up on the outcome parameters was also included in the analysis. Preoperative and postoperative radiological indices (disc height, foraminal height, sagittal angle) were compared within the two groups by using a paired $t$-test. A two-sample $t$-test was performed to compare the changes in the radiological indices from the baseline. Bonforroni technique was used to adjust for multiple comparisons. Correlation between changes in the radiological indices and improvement in the clinical outcome indicators (VAS-back pain and leg pain, ODI, SF-36 scores) was determined using the Pearson's correlation test. A p-value of $<0.05$ was considered as statistically significant.

\section{Results}

\section{Clinical Outcome scores}

\section{1) Oswestry disability index (Fig. 2)}

The mean ODI score for both the Coflex and the comparison group showed significant improvement at six months, one year, and two years as compared to the preoperative score $(p<0.001)$. The mean ODI score in the Coflex group improved from 51.73 preoperatively to 22.91, 22.64 and 17.36 at six months, one year, and two years, respectively. The mean ODI score in the comparison group improved from 49.58 preoperatively to 32.17 ,

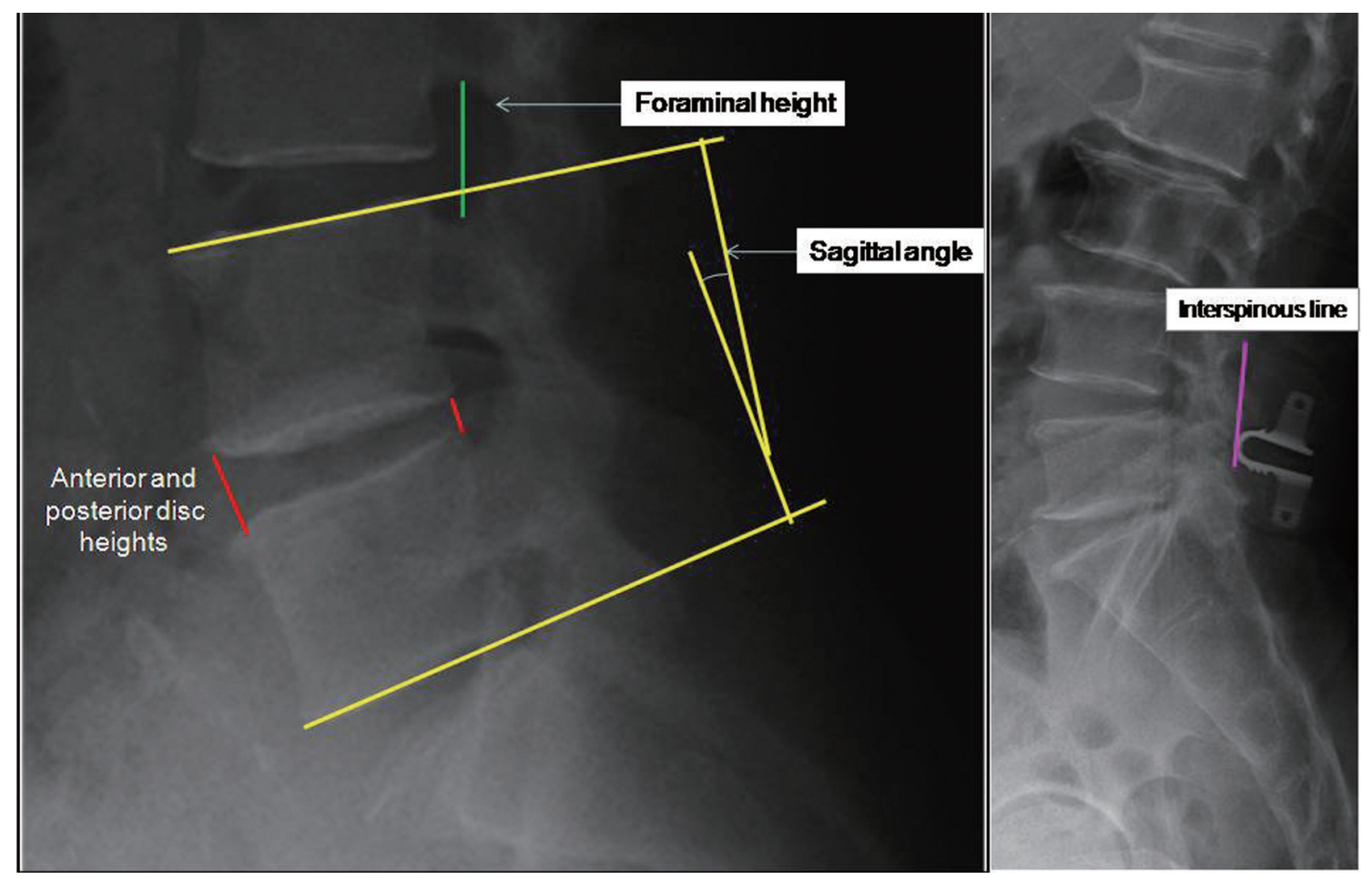

Fig. 1. Lateral lumbosacral radiographs showing radiological indices studied. Red lines, anterior and posterior disc heights; green line, foraminal height; yellow lines, sagittal angle; pink line, interspinous line. 
30.08 , and 28.50 at six months, one year, and two years, respectively. The mean improvement in ODI scores of patients in the Coflex group was significantly greater than the comparison group $(p<0.001)$. The mean difference in ODI improvement between the two groups was 10.4 (95\% confidence interval $[\mathrm{CI}], 8.8-12.1)$, being greater in the Coflex group.

2) VAS-back pain and leg pain (Fig. 3)

The mean VAS score for both back pain and leg pain in

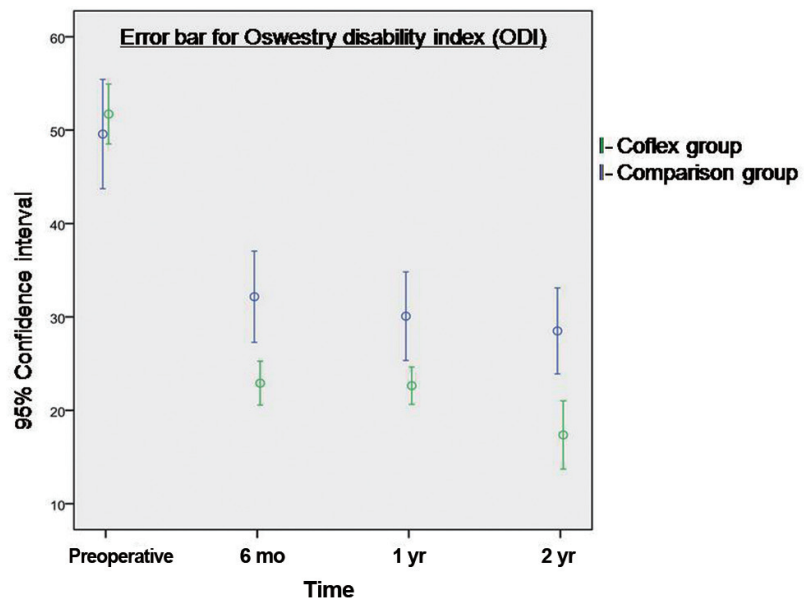

Fig. 2. Comparison of ODI in the two groups at various points in time. There was a significant reduction in ODI scores $(p<0.001)$ compared to baseline in both groups, but reduction in scores in the Ccoflex group was significantly greater than the comparison group $(p<0.001)$. both the Coflex and comparison groups showed a significant improvement at six months, one year and two years as compared to the preoperative score $(p<0.001)$. The mean VAS-back pain score in the Coflex group improved from 60.86 preoperatively to $26.14,13.18$, and 8.39 at six months, one year, and two years, respectively. The mean VAS-back pain score in the comparison group improved from 54.38 preoperatively to $32.88,32.21$, and 29.46 at six months, one year, and two years, respectively.

The mean VAS-leg pain score in the Coflex group improved from 63.45 preoperatively to $19.77,12.50$, and 7.80 at six months, one year, and two years, respectively. The mean VAS-leg pain in the comparison group improved from 57.42 preoperatively to $27.75,28.29$, and 20.92 at six months, one and two years, respectively.

The mean VAS-back pain and mean VAS-leg pain scores in the Coflex group improved significantly greater than the comparison group $(p<0.001)$. The mean difference in VAS-back pain improvement was 17.6 (95\% CI, 14.1-21.1) and in VAS leg pain improvement was 15.5 (95\% CI, 11.2-19.8) between the two groups, each being greater in the Coflex group.

\section{3) SF-36 score}

There was a statistically significant improvement in SF36 scores at six months, one year and two years compared to the preoperative scores in both the Coflex and the comparison groups $(p<0.001)$. The mean SF-36 score in

\section{Emorbar for VAS-backpain}

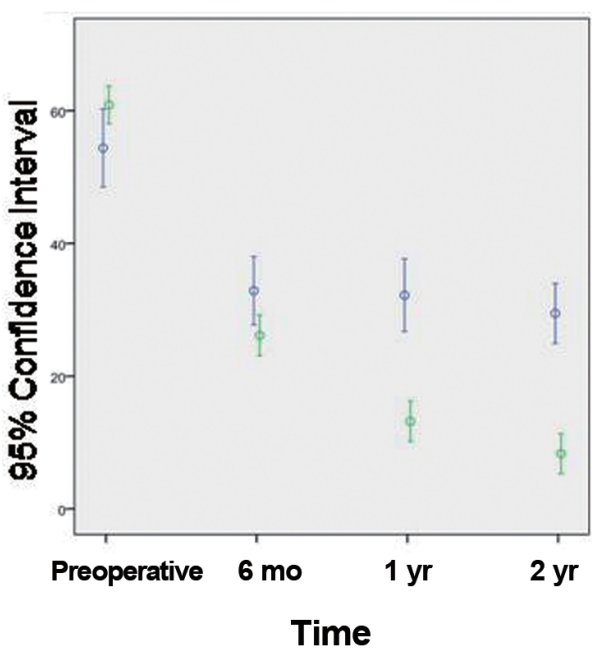

Emorbar forVAS-leg pain

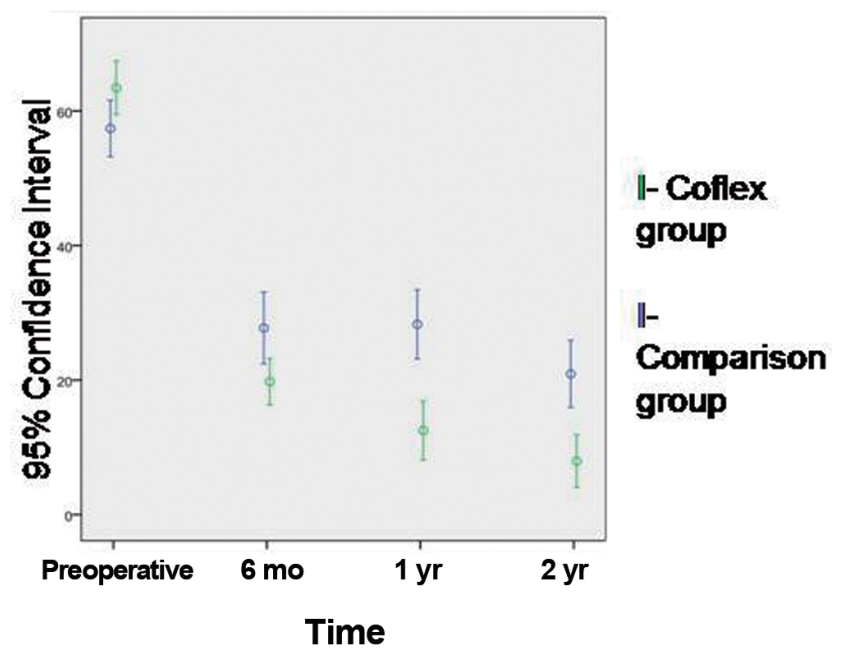

Fig. 3. Comparison of visual analogue scale (VAS)-back pain and leg pain in the two groups at various points in time. There was a significant reduction in VAS-back pain and leg pain scores $(p<0.001)$ compared to baseline in both groups, but reduction in scores in the coflex group was significantly greater than the comparison group $(p<0.001)$. 
the Coflex group improved from 22.91 preoperatively to $51.16,53.60$, and 54.96 at six months, one year, and two years, respectively. The mean SF-36 score in the comparison group improved from 27.33 preoperatively to 43.21 , 47.41, and 49.90 at six months, one year, and two years, respectively. The mean SF-36 scores improved significantly greater in the Coflex group than the comparison group $(p<0.001)$. The mean difference in SF-36 improvement between the two groups was 7.7 (95\% CI, 4.9-10.5), being greater in the Coflex group.

\section{Radiological indices}

\section{1) Coflex group}

There was a significant increase in the mean disc height of $+1.52 \mathrm{~mm}$, at the operated level at six months compared to the preoperative height $(p<0.001)$. However, this increase in height was not sustained at one year and two years follow-up and the resultant height was not significantly greater than the preoperative height (one year: $+0.45 \mathrm{~mm}, p=0.052$; two years: $+0.18 \mathrm{~mm}, p=0.434$ ). The mean foraminal height showed an increase of 3.98 $\mathrm{mm}, 2.44 \mathrm{~mm}$, and $1.76 \mathrm{~mm}$ at the operated level at six months, one year and two years, respectively, which were significantly greater compared to the preoperative level $(p<0.001)$. The mean sagittal angle showed an increase of $4.58^{\circ}, 4.10^{\circ}$, and $3.36^{\circ}$ at six months, one year and two years, respectively, which were significantly greater com-

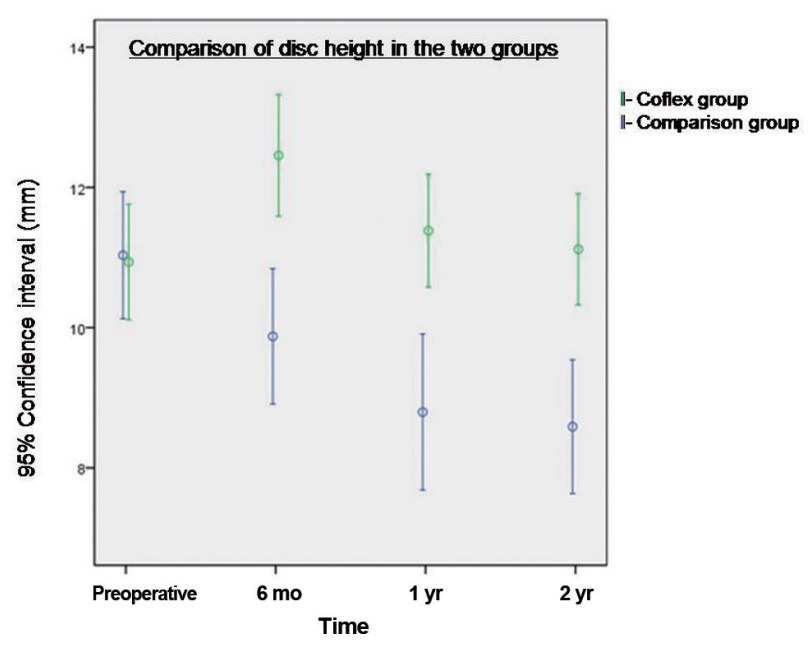

Fig. 4. Comparison of disc heights in the two groups at various points in time. Increase in disc height in the Coflex group was statistically significant $(p<0.001)$ compared to the decrease in height in the comparison group. pared to the preoperative level $(p<0.001)$. Implant subsidence was noted in 14 and 15 implantations at the end of one and two years, respectively, ranging from 1 to $5 \mathrm{~mm}$. However, implant subsidence did not make a significant difference to the clinical outcomes and all patients continued to improve to a comparative degree clinically.

\section{2) Comparison group}

There was a decrease in the mean disc height of 1.16 $\mathrm{mm}, 2.24 \mathrm{~mm}$, and $2.45 \mathrm{~mm}$ at the operated level at six months, one year, and two years, respectively. There was a decrease in the mean foraminal height of $1.63 \mathrm{~mm}, 3.10$ $\mathrm{mm}$, and $3.65 \mathrm{~mm}$ at the operated level at six months, one year and two years, respectively. The mean sagittal angle at the operated level showed an overall increase of $1.92^{\circ}$, $3.30^{\circ}$, and $3.43^{\circ}$ at six months, one year, and two years, respectively. Differences in all the three indices when compared to the preoperative levels, were statistically significant $(p<0.001)$ at six months, one year, and two years.

Preoperative disc height, foraminal height and sagittal angle in both the groups were not significantly different. However, increases in the mean disc and foraminal heights at all three points in time in the Coflex group were significantly greater $(p<0.001)$ than the comparison group (Figs. 4, 5). The increase in the sagittal angle in both the groups was comparable at all points in time (six months: $p=0.932$; one year: $p=0.266$; two years: $p=0.126$ ). Though there were significant differences in the disc and

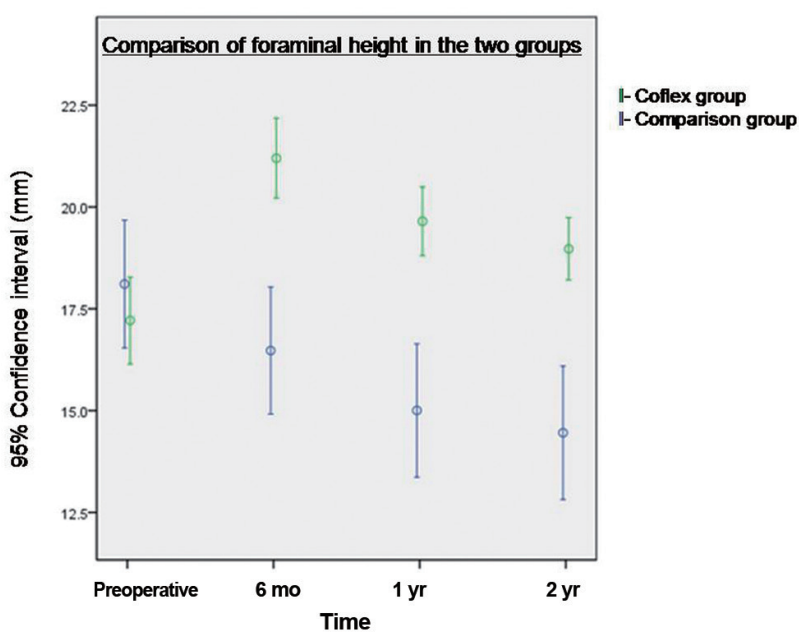

Fig. 5. Comparison of foraminal heights in the two groups at various points in time. There were significant changes in the foraminal height $(p<0.001)$ in both groups compared to baseline. Increase in foraminal height in the Coflex group was statistically significant $(p<0.001)$ compared to the decrease in the comparison group. 
Table 3. Correlation between radiological indices and clinical outcome parameters

\begin{tabular}{|c|c|c|c|c|}
\hline \multirow{3}{*}{$\begin{array}{l}\text { Clinical outcome } \\
\text { parameter }\end{array}$} & \multicolumn{4}{|c|}{ Correlation coefficient $(r) / p$-value } \\
\hline & \multicolumn{2}{|c|}{ Disc height } & \multicolumn{2}{|c|}{ Foraminal height } \\
\hline & Coflex & Comparison & Coflex & Comparison \\
\hline VAS-back pain & $-0.154 / 0.505$ & $-0.196 / 0.359$ & $0.360 / 0.109$ & $-0.068 / 0.753$ \\
\hline VAS-leg pain & $-0.077 / 0.739$ & $0.172 / 0.421$ & $-0.054 / 0.816$ & $0.219 / 0.305$ \\
\hline ODI & $0.214 / 0.352$ & $-0.152 / 0.478$ & $0.028 / 0.904$ & $0.241 / 0.257$ \\
\hline SF-36 & $0.598 / 0.063$ & $-0.242 / 0.255$ & $0.386 / 0.084$ & $-0.034 / 0.874$ \\
\hline
\end{tabular}

VAS, visual analogue scale; ODI, Oswestry disability index; SF-36, short form-36.

foraminal heights after surgery in the two groups, we did not find any correlation between the changes in radiological indices and improvements in the clinical outcome indicators (Table 3).

\section{Complications}

One patient in the Coflex group (dural puncture) and four patients in the comparison group (3-dural puncture and 1-deep infection) had procedure related complications. The incidence of complications in the two groups was not significantly different $(p=0.35)$.

\section{Discussion}

Coflex was first introduced in 1994 by the French orthopedic surgeon Jacques Samani as an alternative to fusion (Samani J, unpublished work). Since then, the device has been used in various degenerative spinal conditions [2630]. However, high level evidence on its clinical efficacy and indications remains limited.

Coflex, a dynamic ISP, reduces the intradiscal pressure and stabilizes the spinal segment in extension, but has almost no effect in flexion, lateral bending and axial rotation in a biomechanical setting [24]. Implantation of Coflex aims at unloading the facet joints, restoring the foraminal height and stabilizing the spinal segment after decompression. Since it can be implanted through the same midline incision, which is used to perform decompression and in view of its proposed benefits, it is worthwhile to determine if additional implantation of Coflex after spinal decompression offers any better clinical outcomes compared to decompression alone. Literature on this issue is very limited.

In a prospective, case-control, one year follow-up study comparing additional implantation of Coflex after decompression to decompression alone for symptomatic LSS, Richter et al. [30] concluded that additional Coflex implantation is safe but not beneficial. They found a significant clinical improvement (VAS, ODI, RolandMorris disability score and pain free walking distance) in patients of both groups after surgery. However, the improvement was not significantly different in the two groups. We performed a prospective, cohort, two year follow-up study comparing the same treatments, in a similar group of patients, using similar clinical outcome indicators (VAS, ODI, and SF-36). Like Richter et al. [30], we did find significant improvement in both the groups compared to the preoperative status. However, patients in the Coflex group in our study improved significantly greater than the comparison group in all clinical outcome indicators at all points in time.

Celik et al. [29] reported clinical outcomes (VAS, ODI) of Coflex implantation after decompression in symptomatic LSS at one year. Patients in their study showed significant clinical improvement after surgery, but they concluded that it was the decompression that was responsible for improvement and additional Coflex implantation was unnecessary. They, however, did not have a comparison group in their study. As it is evident, our findings are in contrast with these authors as well.

Park et al. [27] compared Coflex implantation after spinal decompression with posterior lumbar interbody fusion for degenerative LSS and found comparable clinical effectiveness after either treatments. However, they concluded that the efficacy of Coflex as an adjunct to decompression, may be sustained merely for months after the surgery, and hence, its clinical applications must be reconsidered. Their argument was based on the fact that radiological indices like disc height and reduction of 
vertebral slip were not maintained for long after Coflex implantation. In our study, initial improvement in disc height at six months was not sustained at one year and two years, while improvement in foraminal height and sagittal angle, above the level of significance was sustained at two year follow-up after Coflex. Nevertheless, we found no correlation between the changes in disc and foraminal heights and improvement in the outcome indicators; the Coflex group continued to show significantly greater clinical improvement compared to the comparison group at all points in time in our study. Hence, we believe that the benefit conferred by the additional implantation of Coflex after decompression cannot be presumed to be short- lived only because the changes in the radiological indices are not sustained over time.

In summary, like other authors, our study showed that surgical treatment offers significant improvement in the clinical outcomes in symptomatic LSS $[27,29,30]$. However, the Coflex group in our study improved significantly greater than the comparison group, and this is not in agreement with the only other study similar to ours [30]. Since we did not find any correlation between the changes in radiological indices and improvement in outcomes, we do not agree with the other authors that Coflex implantation does not offer added benefit [29] or that the benefit may not be sustained for a long time [27]. Implantation of Coflex as an adjunct to decompression is superior to decompression alone as it offers significantly better clinical outcomes in patients with symptomatic LSS. The superior clinical outcomes with Coflex implantation can be attributed to its function of unloading the facet joints and stabilizing the spinal segment after decompression. Moreover, the technique of implanting the device is simple and can be done through the same midline approach that is used for decompression.

While a smaller sample size is a limitation of our study, we firmly believe that the study assumes relevance because of its prospective nature and the findings that are different from what has been previously reported. Randomization of patients in the two groups would have further strengthened the study. However, the decision to receive Coflex was left to the patients in our study. The demographic data in the two groups was comparable and bias was further minimized as all the patients were operated by the same surgeon (N.K.). Stronger conclusions can be drawn from a similar study with greater number of patients and a longer follow-up, which is currently under way.

\section{Conclusions}

To conclude, additional Coflex implantation after spinal decompression in symptomatic LSS offers better clinical outcomes than decompression alone at short- term. Changes in radiological indices do not correlate with the improvements in clinical outcomes after surgery for symptomatic LSS.

\section{Conflict of Interest}

No potential of conflict of interest relevant to this article was reported.

\section{References}

1. Lin SI, Lin RM, Huang LW. Disability in patients with degenerative lumbar spinal stenosis. Arch Phys Med Rehabil 2006;87:1250-6.

2. Fraser JF, Huang RC, Girardi FP, Cammisa FP Jr. Pathogenesis, presentation, and treatment of lumbar spinal stenosis associated with coronal or sagittal spinal deformities. Neurosurg Focus 2003;14:e6.

3. Seong YJ, Lee JS, Suh KT, Kim JI, Lim JM, Goh TS. Posterior decompression and fusion in patients with multilevel lumbar foraminal stenosis: a comparison of segmental decompression and wide decompression. Asian Spine J 2011;5:100-6.

4. Ahmed M, Modic MT. Neck and low back pain: neuroimaging. Neurol Clin 2007;25:439-71.

5. Ogikubo O, Forsberg L, Hansson T. The relationship between the cross-sectional area of the cauda equina and the preoperative symptoms in central lumbar spinal stenosis. Spine (Phila Pa 1976) 2007;32:1423-8.

6. Sigmundsson FG, Kang XP, Jonsson B, Stromqvist B. Prognostic factors in lumbar spinal stenosis surgery. Acta Orthop 2012;83:536-42.

7. Anjarwalla NK, Brown LC, McGregor AH. The outcome of spinal decompression surgery 5 years on. Eur Spine J 2007;16:1842-7.

8. Athiviraham A, Yen D. Is spinal stenosis better treated surgically or nonsurgically? Clin Orthop Relat Res 2007;458:90-3.

9. Malmivaara A, Slatis P, Heliovaara M, et al. Surgical or nonoperative treatment for lumbar spinal stenosis? A randomized controlled trial. Spine (Phila Pa 1976) 2007;32:1-8. 
10. Mariconda M, Fava R, Gatto A, Longo C, Milano C. Unilateral laminectomy for bilateral decompression of lumbar spinal stenosis: a prospective comparative study with conservatively treated patients. J Spinal Disord Tech 2002;15:39-46.

11. Weinstein JN, Tosteson TD, Lurie JD, et al. Surgical versus nonsurgical therapy for lumbar spinal stenosis. N Engl J Med 2008;358:794-810.

12. Spivak JM. Degenerative lumbar spinal stenosis. J Bone Joint Surg Am 1998;80:1053-66.

13. Sengupta DK, Herkowitz HN. Degenerative spondylolisthesis: review of current trends and controversies. Spine (Phila Pa 1976) 2005;30(6 Suppl):S71-81.

14. Yone K, Sakou T, Kawauchi Y, Yamaguchi M, Yanase M. Indication of fusion for lumbar spinal stenosis in elderly patients and its significance. Spine (Phila Pa 1976) $1996 ; 21: 242-8$.

15. Kim MC, Chung HT, Kim DJ, Kim SH, Jeon SH. The clinical and radiological outcomes of minimally invasive transforaminal lumbar interbody single level fusion. Asian Spine J 2011;5:111-6.

16. Chung SS, Lee CS, Kim SH, Chung MW, Ahn JM. Effect of low back posture on the morphology of the spinal canal. Skeletal Radiol 2000;29:217-23.

17. Fujiwara A, An HS, Lim TH, Haughton VM. Morphologic changes in the lumbar intervertebral foramen due to flexion-extension, lateral bending, and axial rotation: an in vitro anatomic and biomechanical study. Spine (Phila Pa 1976) 2001;26:876-82.

18. Penning L, Wilmink JT. Posture-dependent bilateral compression of L4 or L5 nerve roots in facet hypertrophy. A dynamic CT-myelographic study. Spine (Phila Pa 1976) 1987;12:488-500.

19. Verbiest H. A radicular syndrome from developmental narrowing of the lumbar vertebral canal. J Bone Joint Surg Br 1954;36:230-7.

20. Willen J, Danielson B, Gaulitz A, Niklason T, Schonstrom N, Hansson T. Dynamic effects on the lumbar spinal canal: axially loaded CT-myelography and MRI in patients with sciatica and/or neurogenic claudication. Spine (Phila Pa 1976) 1997;22:2968-76.

21. Richards JC, Majumdar S, Lindsey DP, Beaupre
GS, Yerby SA. The treatment mechanism of an interspinous process implant for lumbar neurogenic intermittent claudication. Spine (Phila Pa 1976) 2005;30:744-9.

22. Lindsey DP, Swanson KE, Fuchs P, Hsu KY, Zucherman JF, Yerby SA. The effects of an interspinous implant on the kinematics of the instrumented and adjacent levels in the lumbar spine. Spine (Phila $\mathrm{Pa}$ 1976) 2003;28:2192-7.

23. Kettler A, Drumm J, Heuer F, et al. Can a modified interspinous spacer prevent instability in axial rotation and lateral bending? A biomechanical in vitro study resulting in a new idea. Clin Biomech (Bristol, Avon) 2008;23:242-7.

24. Wilke HJ, Drumm J, Haussler K, Mack C, Steudel WI, Kettler A. Biomechanical effect of different lumbar interspinous implants on flexibility and intradiscal pressure. Eur Spine J 2008;17:1049-56.

25. Bono CM, Vaccaro AR. Interspinous process devices in the lumbar spine. J Spinal Disord Tech 2007;20:255-61.

26. Kong DS, Kim ES, Eoh W. One-year outcome evaluation after interspinous implantation for degenerative spinal stenosis with segmental instability. J Korean Med Sci 2007;22:330-5.

27. Park SC, Yoon SH, Hong YP, Kim KJ, Chung SK, Kim HJ. Minimum 2-year follow-up result of degenerative spinal stenosis treated with interspinous $\mathrm{u}$ (coflex). J Korean Neurosurg Soc 2009;46:292-9.

28. Cabraja M, Abbushi A, Woiciechowsky C, Kroppenstedt $\mathrm{S}$. The short- and mid-term effect of dynamic interspinous distraction in the treatment of recurrent lumbar facet joint pain. Eur Spine J 2009;18:1686-94.

29. Celik H, Derincek A, Koksal I. Surgical treatment of the spinal stenosis with an interspinous distraction device: do we really restore the foraminal height? Turk Neurosurg 2012;22:50-4.

30. Richter A, Schutz C, Hauck M, Halm H. Does an interspinous device (Coflex) improve the outcome of decompressive surgery in lumbar spinal stenosis? One-year follow up of a prospective case control study of 60 patients. Eur Spine J 2010;19:283-9. 